\title{
Cardiac Tamponade Associated with Blunt Cardiac Injury: Its Definitive Management in the Emergency Department
}

\author{
Tetsuya Yumoto ${ }^{*}$, Nao Umei ${ }^{1}$, Yo Kinami ${ }^{1}$, Hideo Yamanouchi' ${ }^{1}$ Keiji Sato ${ }^{1}$, Yosuke Ueno ${ }^{2}$, \\ Yosuke Kuroko², Zenichi Masuda², Toyomu Ugawa' ${ }^{1}$, Yoshihito Ujike1 \\ ${ }^{1}$ Advanced Emergency and Critical Care Medical Center, Okayama University Hospital, Okayama, Japan \\ ${ }^{2}$ Department of Cardiovascular Surgery, Okayama University Graduate School of Medicine, Dentistry, and \\ Pharmaceutical Sciences, Okayama, Japan \\ Email: tyumoto@cc.okayama-u.ac.jp
}

Received 30 June 2015; accepted 24 July 2015; published 27 July 2015

Copyright (C) 2015 by authors and Scientific Research Publishing Inc.

This work is licensed under the Creative Commons Attribution International License (CC BY). http://creativecommons.org/licenses/by/4.0/

(c) (i) Open Access

\begin{abstract}
Cardiac tamponade secondary to blunt cardiac injury is an extremely serious and life-threatening condition that the emergency physician is required to make definitive and appropriate management and resuscitation. Although blunt cardiac injury includes a wide spectrum of pathological conditions, cardiac tamponade due to blunt trauma is rarely seen because most of the patients with cardiac rupture die at the scene or before arrival at the hospital. Definitive surgical repair is commonly necessary following pericardial decompression if the patient is too unstable with impending cardiac arrest. Bringing the patient to definitive surgery as soon as possible is crucial for the chance of survival to the emergency physician. We describe two cases of cardiac tamponade resulting from blunt chest trauma successfully treated with a definitive management and surgical repair.
\end{abstract}

\section{Keywords}

Blunt Cardiac Injury, Cardiac Tamponade, Definitive Management

\section{Introduction}

Cardiac tamponade which is associated with blunt cardiac injury is an extremely serious and life-threatening condition that requires emergency physicians to immediately initiate appropriate management strategies prior to emergency surgery. We describe two instructive cases of cardiac tamponade associated with blunt chest trauma.

\footnotetext{
${ }^{*}$ Corresponding author.
}

How to cite this paper: Yumoto, T., Umei, N., Kinami, Y., Yamanouchi, H., Sato, K., Ueno, Y., Kuroko, Y., Masuda, Z., Ugawa, T. and Ujike, Y. (2015) Cardiac Tamponade Associated with Blunt Cardiac Injury: Its Definitive Management in the Emergency Department. Open Journal of Emergency Medicine, 3, 9-12. http://dx.doi.org/10.4236/ojem.2015.32002 


\section{Case 1}

A 61-year-old female was transferred from another hospital after presenting with cardiac tamponade after a motor vehicle crash. On arrival at the other hospital, the patient was unconscious, her blood pressure was not recordable, and she had a heart rate of 82 beats/min (bpm). A focused assessment with sonography for trauma (FAST) detected pericardial effusion. The patient was referred to our tertiary hospital followed by pericardiocentesis. Minor traumatic subarachnoid hemorrhaging, traumatic dissection of the descending aorta, and liver injuries that did not require urgent surgical intervention were also identified.

On arrival at our hospital (nearly two hours after the accident), the patient's blood pressure was $60 / 40 \mathrm{mmHg}$, and her heart rate was $160 \mathrm{bpm}$ under noradrenaline administration. The clogging of a pericardial catheter revealed that the patient was hemodynamically unstable, so we performed anemergency subxiphoidpericardiotomyin the emergency room (ER) whilst preparations were made for an emergency thoracotomy. Further pericardial decompression resulted in an increase in the patient's systolic blood pressure to over $80 \mathrm{mmHg}$; however, massive uncontrollable bleeding of bright red blood from the pericardial sac occurred, which was suggestive of a left atrial or ventricular injury. The patient was rushed to the operating room (OR) nearly one hour after her arrival. A left atrial appendage tear (length: approximately $3 \mathrm{~cm}$ ) extending to the base of the heart was found and was successfully repaired under cardiopulmonary bypass (CPB). Sixty units of red blood cell concentrate (RBC) were required within the first 24 hours after the patient's arrival.

The patient was successfully weaned off ventilation on day 24 and was transferred to another hospital to undergo rehabilitation on hospital day 58 when her Glasgow Outcome Scale was 4 with moderate disability.

\section{Case 2}

A 36-year-old female was transferred to our tertiary hospital 50 minutes after a high-speed car crash. On arrival, she was pale and distressed and had a heart rate of $163 \mathrm{bpm}$ and a blood pressure of 63/55 mmHg. Pericardial effusion was detected with FAST after she presented with cardiac tamponade. The patient was immediately referred to a cardiovascular surgeon. Tracheal intubation was performed, and an arterial line was quickly installed to enable careful monitoring. The patient's heart rate and systolic blood pressure remained above $150 \mathrm{bpm}$ and $60 \mathrm{mmHg}$, respectively, after fluid resuscitation and dopamine administration. After taking the patient's unchanged vital signs and the time elapsed since the accident into consideration, we decided to perform pericardial drainage via pericardial decompression in the ER due to a concern about uncontrollable hemorrhaging, as the patient collapsed whenever she attempted to stand. A contrast-enhanced computed tomography scan revealed pericardial effusion and the extravasation of contrast medium from at least two portions of the right atrium (Figure 1). In addition, liver and right kidney injuries were identified. The patient was moved to the OR as soon

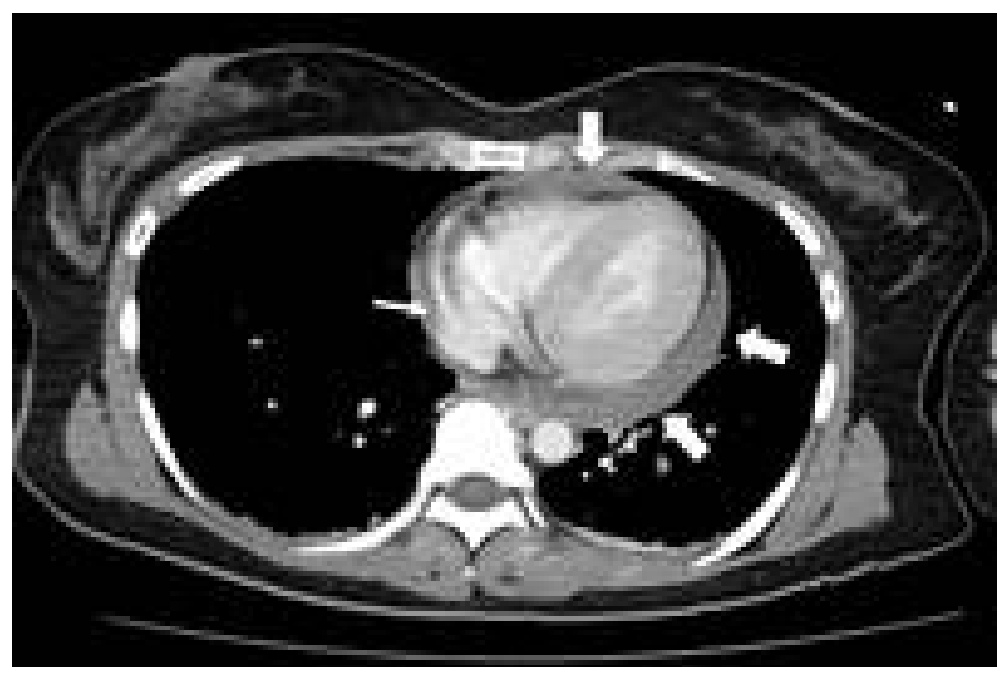

Figure 1. Contrast-enhanced computed tomography shows contrast extravasation from right atrium (thin arrow) and pericardial effusions (thick arrow). 
as possible (approximately one hour after the patient's arrival). A median sternotomy was performed followed by pericardiotomy, which raised the patient's systolic blood pressure to over $150 \mathrm{mmHg}$. Two tears of $5 \mathrm{~mm}$ in length were found in the right atrial appendage, which were successfully repaired without $\mathrm{CPB}$. The patient required four units of RBC within 24 hours of her arrival. Her postoperative course was uneventful, and she was discharged on day 22.

\section{Discussion}

Cardiac tamponade resulting from blunt cardiac injury is an extremely life-threatening and time-critical emergency condition and requires prompt management and definitive surgical repair [1]-[6]. It is associated with cardiac rupture; tears in the vena cava; or, in rare cases, injuries to the coronary artery in patients with preserved pericardia [4]. Although a study of an autopsy series found that the incidence of cardiac rupture did notdiffer among the four chambers of the heart, patients that suffer ruptures involving the right chambers exhibit ahigher survival rate [5] [6].

FAST can be used to differentiate between pericardial effusion and other causes of shock, such as massive hemothorax or intraperitoneal hemorrhaging [7]. Although the use of echocardiography has become widespread, clinicians should pay careful attention to patients with subcutaneous emphysema or pneumothorax, as echocardiography exhibits low sensitivity for detecting pericardial effusion in such patients. Also, pericardial effusion is only detectable after a sufficient amount of pericardial bleeding has occurred. Thus, careful and repetitive investigations are required to obtain an accurate diagnosis [2].

Secondary, we discuss how to manage cases in which blunt cardiac rupture is highly suspected after echocardiography in patients with recordable vital signs. Since definitive surgical repair is essential, we first need to refer the patient to the operating team [2] [5]. Although Western Trauma association has published a guideline for the resuscitative thoracotomy, it will depend on the multiple factors such as personnel and situations [8]. While the OR is being prepared, pericardial decompression should be performed either by pericardiocentesis or pericardiotomy in cases involving impending cardiac arrest. Pericardiocentesis can be effective atrelievingcardiac tamponade before definitive surgical management. However, as it is a temporary procedure subsequentblood clotting can render it ineffective, as occurred in case 1 [3]. On the other hand, subxiphoid pericardiotomy is a more effective method for decompressing the pericardium and can be performed within a few minutes and without specialized instruments [9]. However, it can cause uncontrollable bleeding due to major cardiac tears, as in case 1. If the patient is hemodynamically unstable, e.g., exhibits bradycardia or their blood pressure is not recordable, and it is expected that they face a prolonged wait for definitive surgical management, emergency physicians should perform pericardial drainage or resuscitative thoracotomy without hesitation in the ER. In case 2, the patient's blood pressure was sustained with catecholamine support while the OR was set up, which resulted in a minimal blood transfusion requirement. Emergency physicians should select appropriate management strategies based on a variety of factors such as the time period that has elapsed since the injury, changes in the patient's vital signs, and the time until surgery [10].

\section{Conclusion}

In conclusion, the initiation of appropriate management strategies by emergency physicians and cooperation with surgical teams are important for treating patients who present with cardiac tamponade due to blunt cardiac injury.

\section{References}

[1] Courcy, P.A., Stair, T.O. and Brotman, S. (1984) Subxiphoid Pericardial Window in Traumatic Pericardial Tamponade. The American Journal of Emergency Medicine, 2, 153-155. http://dx.doi.org/10.1016/S0735-6757(84)80010-8

[2] Nan, Y.Y., Lu, MS., Liu, K.S., Huang, Y.K., Tsai, F.C., Chu, J.J. and Lin, P.J. (2009) Blunt Traumatic Cardiac Rupture: Therapeutic Options and Outcomes. Injury, 40, 938-945. http://dx.doi.org/10.1016/j.injury.2009.05.016

[3] Lee, T.H., Ouellet, J.F., Cook, M., Schreiber, M.A. and Kortbeek, J.B. (2013) Pericardiocentesis in Trauma: A Systemic Review. The Journal of Trauma and Acute Care Surgery, 75, 543-549. http://dx.doi.org/10.1097/TA.0b013e3182a1fea2

[4] Fulda, G., Brathwaite, C.E., Rodriguez, A., Turney S.Z., Dunham, C.M. and Cowley, R.A. (1991) Blunt Traumatic 
Rupture of the Heart and Pericardium: A Ten-Year Experience (1979-1989). The Journal of Trauma, 31, 167-172. http://dx.doi.org/10.1097/00005373-199131020-00003

[5] Namai, A., Sakurai, M. and Fujiwara, H. (2007) Five Cases of Blunt Traumatic Cardiac Rupture: Success and Failure in Surgical Management. General Thoracic and Cardiovascular Surgery, 55, 200-204. http://dx.doi.org/10.1007/s11748-007-0106-x

[6] Pevec, W.C., Udekwu, A.O. and Peitzman, A.B. (1989) Blunt Rupture of the Myocardium. The Annals of Thoracic Surgery, 48, 139-142. http://dx.doi.org/10.1016/0003-4975(89)90203-8

[7] Karalis, D.G., Victor, M.E., Davis, G.A., McAllister, M.P., Covalesky, V.A., Ross Jr., J.J., Foley, R.V., Kerstein, M.D. and Chandrasekaran, K. (1994) The Role of Echocardiography in Blunt Chest Trauma: A Transthoracic and Transesophageal Echocardiographic Study. The Journal of Trauma, 36, 53-58. http://dx.doi.org/10.1097/00005373-199401000-00008

[8] Burlew, C.C., Moore, E.E., Moore, F.A., Coimbra, R., Mclntyre Jr., R.C., Davis, J.W., Sperry, J. and Biffl, W.L. (2012) Western Trauma Association Critical Decisions in Trauma: Resuscitative Thoracotomy. The Journal of Trauma and Acute Care Surgery, 73, 1359-1363. http://dx.doi.org/10.1097/TA.0b013e318270d2df

[9] Kurimoto, Y., Hase, M., Nara, S., Yama, N., Kawaharada, N., Morishita, K., Higami, T. and Asai, Y. (2006) Blind Subxiphoid Pericardiotomy for Cardiac Tamponade Because of Acute Hemopericardium. The Journal of Trauma, 61, 582-585. http://dx.doi.org/10.1097/01.ta.0000236060.37952.ce

[10] Fitzgerald, M., Spencer, J., Johnson, F., Marasco, S., Atkin, C. and Kossmann, T. (2005) Definitive Management of Acute Cardiac Tamponade Secondary to Blunt Trauma. Emergency Medicine Australasia, 17, 494-499. http://dx.doi.org/10.1111/j.1742-6723.2005.00782.x 